\title{
ESTRATÉGIAS DE REDUÇÃO DE DANOS EM ESTUDANTES UNIVERSITÁRIOS QUE FAZEM USO DE DROGAS
}

\author{
João Filippini
}

Dissertação apresentada ao Programa de Pós-graduação em Psicologia da Faculdade de Filosofia, Ciências e Letras de Ribeirão Preto da Universidade de São Paulo, para obtenção do título de Mestre em Ciência. Área de concentração: Psicologia em Saúde e Desenvolvimento.

Orientadora: Clarissa Mendonça Corradi-Webster 


\section{RESUMO}

Filippini, J.D. (2020). Estratégias de redução de danos entre estudantes universitários que fazem uso de drogas (Dissertação de Mestrado). Programa de Pós-Graduação em Psicologia, Faculdade de Filosofia, Ciências e Letras de Ribeirão Preto, Universidade de São Paulo, Ribeirão Preto - SP.

A literatura aponta que o uso de álcool e outras drogas entre universitários é uma questão de grande relevância pelo fato de que ocorre um consumo mais intenso nessa fase da vida. Esse consumo em maior escala está associado a comportamentos de risco e consequências negativas. Faz-se necessário a formulação de estratégias de redução de danos para que esses comportamentos e consequências possam diminuir de intensidade e frequência, reduzindo os prejuízos decorrentes do consumo. No contexto universitário existem poucos estudos que examinam comportamentos de redução de danos, tornando necessário um melhor exame desses. O objetivo deste estudo é compreender percepções de estudantes universitários que fazem uso de álcool e outras drogas sobre seu consumo e quais suas estratégias de redução de danos para se proteger de possíveis prejuízos decorrentes de seu uso, e, mais especificamente, compreender como o modo de vida universitário pode tornar o jovem mais vulnerável em seu consumo de substâncias e conhecer o processo de construção das estratégias de redução de danos desenvolvidas pelos estudantes. Para tal, foram realizadas entrevistas com quarenta e quatro estudantes no formato de grupos focais. Os participantes foram convidados a partir do método "snowball", e após receberem o convite do pesquisador lhes foi pedido que estendessem o convite a conhecidos que poderiam contribuir com a pesquisa, tornando os grupos um ambiente mais próximo de sua rede social. Ocorreram oito grupos, variando entre quatro e oito participantes, sendo um total de dezenove mulheres e vinte e cinco homens. Todos preencheram o AUDIT auto-aplicável e obtiveram escores entre 7 e 26, com uma média de 13,5, indicando um padrão médio de uso de risco, mantendo esse padrão quando responderam ao ASSIST, principalmente para ácool, tabaco e maconha. Dentre as consequências negativas que mais frequentemente citadas pelos participantes estão a maior probabilidade de sofrerem danos físicos e sociais, e perda de memória, além de prejuízos financeiros e acadêmicos. Ao levantar essas consequências, descrevem que o que as torna negativas geralmente se relaciona com a perda de autocontrole ao usar a substância, e entendem que as situações de risco também giram em torno de não ter o controle do que pode acontecer, principalmente em festas "open bar". A partir dessa percepção, ressaltam um sentimento de vulnerabilidade em seu consumo. Quanto às suas estratégias de redução de danos para lidar com as questões descritas acima estão: beber água enquanto consome drogas, se alimentar antes de fazer o consumo, fazer esse consumo acompanhado de pessoas de confiança, não dirigir quando for realizar consumo exagerado, e buscar conversar com amigos e usar de sua rede de apoio social para proteção nesse sentido. Também deve ser ressaltada a diferença do nível de preocupação com o autocuidado entre estudantes dependendo de sua idade e ano no curso, sendo os alunos mais novos os que menos se preocupam em reduzir seus danos. Conforme se desenvolvem no curso, usam de suas experiências com o consumo e de seu aprendizado com os amigos da rede de apoio para desenvolver e manter estratégias de redução de danos. O projeto foi aprovado pelo Comitê de Ética em abril e 2018.

Palavras-chave: Uso de drogas; Universitários; Redução de Danos 


\begin{abstract}
Filippini, J.D. (2020). Damage reduction strategies among university students who use drugs (Master Thesis). Post-Graduation Program of Psychology, Ribeirão Preto College of Philosophy, Sciences and Literature, University of São Paulo, Ribeirão Preto - SP.
\end{abstract}

Literature points out that alcohol consumption and other drugs among university students is a question of extreme matter, since the consumption during this stage of life is the highest. This consumption in higher doses is associated with risk behaviors and negative consequences. To decrease these behaviors and consequences in intensity and frequency, the formulation of damage reducing strategies is needed, also lowering the hazards of consumption. In the university scenario, there are few studies that examine behaviors of damage reduction, making a better analysis of great importance. The objective of this study is to comprehend perceptions of university students that consume alcohol and other drugs on their consumption and what their damage reduction strategies are to protect themselves from possible injuries they may encounter due to their use, and more specifically, comprehend how the university lifestyle can make the youth more vulnerable to consumption of substances and to know the process behind the construction of damage reduction strategies developed by students. For such, interviews with forty-four students were realized in focal group format. The participants were invited through the "snowball" method, after receiving the invitation from the researcher, they were asked to invite fellow colleagues that could contribute to the research, making the groups a close friendly environment. Eight groups were analyzed, varying between four and eight participants, totaling nineteen women and twenty-five men. All of them filled out the self-applied AUDIT and achieved scores between 7 and 26, with a mean score of 13,5 , indicating a medium standard risk of use, maintaining this standard when answering to the ASSIST, specially for alcohol, tobacco and marijuana. Among the most mentioned negative consequences by patients are a higher probability to suffer physical and social damage, memory loss, aside from the financial and learning loss. Bringing these consequences to mind, one can see what turns them negative is generally related to the loss of auto control when using the substance, and understand that the risk situations are also related to losing the control of what can happen, specially at "open bar" parties. From this perspective, a feeling of vulnerability of consumption stands out. As for their damage reduction strategies to deal with the questions described above there are: drinking water while consuming drugs, eating before the use, consuming along trustworthy people, not driving when consuming in excess, and talking with friends to build a trust zone for protection in this sense. The difference in level of worry with selfcare between students should be brought depending on their age and year of entrance in the university, being the youngest the ones who worry less with damage reduction. As the students develop in their courses, they use their experiences with consumption and learnings with friends from their trust zone to develop and maintain damage reduction strategies. The project has been approved by the Committee of Ethics on April of 2018.

Key words: Drug use; University Students; Harm Reduction 


\section{INTRODUÇÃO}

Para a contextualização do objetivo do presente estudo, que busca compreender as percepções sobre redução de danos por parte dos universitários que fazem uso de álcool e outras drogas, dividiu-se a introdução teórica em três tópicos: primeiramente um panorama geral do uso de substâncias entre universitários, seguido de uma conceitualização dos princípios relacionados à redução de danos, finalizando com uma contextualização de como a redução de danos se apresenta no ambiente universitário.

No tópico inicial busca-se também explicitar as possíveis consequências negativas decorrentes do consumo indevido ou exagerado, estabelecendo uma relação com os objetivos buscados pela redução de danos, demonstrando como a última se mostra necessária, e como esses objetivos podem permear o consumo, diminuindo as chances de problemas acarretados pelo mesmo dentro do contexto de uma universidade.

\subsection{Uso de Substâncias entre Estudantes Universitários}

O uso de drogas é caracterizado como sendo a auto-administração de substâncias psicoativas realizada por um indivíduo (World Health Organization, 1994), sendo que os padrões dessa autoadministração levam à caracterização do uso abusivo e da dependência. $\mathrm{O}$ uso abusivo pode ser descrito como um uso que leva o indivíduo a situações de risco, problemas legais ou o impossibilita de realizar obrigações importantes (APA, 2000). A dependência se dá quando esse uso é mantido e procurado independentemente das consequências às quais leva (WHO, 1994). Considera-se que os que fazem uso de substâncias o fazem com o intuito de obter benefícios através de seus efeitos, por proporcionarem prazer ou por diminuírem qualquer sensação dolorosa (WHO, 2004).

O uso de substâncias é recorrente ao longo da história da humanidade, sendo, mais recentemente, uma questão a qual é dada maior atenção. De acordo com o II Levantamento Nacional Sobre o Uso Domiciliar de Substâncias Psicoativas, aproximadamente 22,8\% da população brasileira, entre 12 e 65 anos, já fez uso, em algum momento da vida, de alguma substância psicotrópica. O álcool e o tabaco não estão inclusos nessa porcentagem, uma vez que foram avaliados à parte, e atingiram uma estimativa de 74,6\%, e 44\%, respectivamente (Carlini et al., 2007). Deve-se também observar essa conjuntura nacional dentro de um cenário mundial em que o consumo geral dessas substâncias vem aumentando (UNODCO, 2004).

Acredita-se que o início do consumo de substâncias ocorre nas fases da adolescência (Soldera, et al., 2004) e na juventude. Esse consumo parece se exacerbar entre universitários (Peuker, Fogaça \& Bizzaro, 2006). Na adolescência, é observado no Brasil que 25,5\% dos 
adolescentes (idade mínima de 10 anos) relatam terem feito uso em algum momento na vida (Carlini et al., 2010). Já entre jovens estudantes universitários, temos os escores de uso na vida de $82,6 \%$ de uso de álcool, 46,7\% de uso de derivados de tabaco, e 48,7\% de uso de substâncias ilícitas, dentre essas temos a maconha com 26,1\%, o ecstasy com 7,5\%, e os alucinógenos (LSD) com 7,6\% (SENAD, 2010). Podemos observar, a partir desses dados, que o uso de substâncias, apesar de se iniciar na adolescência, se intensifica ao longo da vida, mostrando um intenso aumento na fase da juventude, principalmente no momento e ambiente universitários. Derefinko et al. (2016) acompanharam a trajetória de uso de drogas dos últimos anos de ensino médio e nos primeiros anos de ensino superior de jovens estudantes, demonstrando que, principalmente no primeiro ano de faculdade, há um aumento significativo do uso de álcool, maconha, e drogas pesadas, tanto de indivíduos que faziam pouco uso, apenas estavam experimentando, e que já faziam um uso pesado das substâncias.

O ingresso na universidade muitas vezes representa o primeiro momento de maior independência na vida do indivíduo, que em muitos casos sai de sua cidade e da casa da família para viver em outro contexto com novas descobertas, caracterizando um momento de vulnerabilidade pelas mudanças que ocorrem em sua vida, além também de um momento em que o mesmo tem maior liberdade para tomar suas decisões. Dentro dessa dinâmica, é possível afirmar que o afastamento da casa da família pode ser um preditor para o aumento no uso de substâncias, tendo a isso somadas as mudanças no espaço de sociabilidade e inserção social, em que o uso é estimulado, podendo levar a um isolamento social de quem tem atitudes contrárias (Laranjo \& Soares, 2006). Além disso, dentro do ambiente universitário, o contato e as amizades com pessoas que fazem uso de alguma substância, como álcool, maconha, ou outras, pode motivar o indivíduo a experimentar e começar a fazer uso também (Dennhardt \& Murphy, 2013).

Percebe-se então que ocorre uma relação de expectativas positivas quanto ao consumo de álcool, havendo maior propensão a esse comportamento nessa etapa da vida (Fachini \& Furtado, 2013; Lange, et al., 2002). Em contraponto a isso, não existe nas universidades um espaço ou uma abertura para que se converse sobre o consumo de álcool, levando a uma dificuldade de acesso a informações de prevenção e modos de uso menos nocivos (Amaral, Lourenço e Ronzani, 2006).

Dados quantitativos corroboram esse fato tanto para o álcool quanto para outras substâncias, como demonstrado no I Levantamento Nacional Sobre o Uso de Álcool, Tabaco e Outras Drogas entre Estudantes Universitários de 27 Capitais Brasileiras, em que os estudantes apresentaram não somente um alto índice de uso na vida, mas também nos últimos 12 meses e nos últimos 30 dias. A substância mais usada é o álcool, seguido de drogas ilícitas, e, em terceiro, pelo tabaco (SENAD, 2010). 
Para Kutsche et al. (2006) as principais motivações para o uso de álcool podem ser divididas em dois tipos: a de busca dos benefícios que seu consumo pode produzir, e a de copia de um comportamento social. Para os autores, esses benefícios giram em torno de uma maior desenvoltura social e extroversão, facilitando as interações com outras pessoas, e a copia de comportamento busca uma diminuição da dificuldade em se lidar com sintomas ansiosos e neuróticos.

As razões apontadas para o consumo de álcool nessa faixa etária variam, mas, em sua maioria, giram em torno de razões sociais, como uma facilitação para a interação social, melhoria de eventos e reuniões sociais, ajudar a entrar em um "clima de festa", dentre outros, sendo estas razões mais associadas a um uso moderado da substância, não estando tão relacionadas a um uso pesado (Kuntsche et al., 2005). Pensando-se no cenário universitário como também um espaço para a interação social em que a ocorrência de festas e eventos sociais do tipo são comuns e tem alta frequência, consegue-se então perceber as motivações para um uso recorrente do álcool entre as pessoas inseridas nesse meio.

Apesar de ser a substância de maior consumo nas estatísticas, o aumento do uso nessa etapa da vida não se restringe somente ao álcool, como demonstra o estudo de Silva, Rego, Roque \& Valente (2014). Neste estudo, que se trata de um levantamento de dados quantitativos feito em uma universidade pública brasileira, é possível ver que o primeiro encontro com as substâncias se dá, em geral, antes da entrada na universidade, mas há um acentuado aumento no uso nos primeiros anos, seguido de uma diminuição nos últimos anos de curso. Esse aumento não se relaciona meramente ao álcool, mas a todas as substâncias. Ainda assim, ao se estudar uso de substâncias nesse contexto, como em diferentes outros contextos, há uma predileção pelo álcool acima das outras substâncias, pelo seu amplo uso (Goldsmith et al., 2011).

A maconha, por exemplo, apresenta uma prevalência de uso nos últimos 12 meses de 13,8\% na população universitária (SENAD, 2010). Dentre as razões estudadas para o uso de maconha, pode-se destacar, primeiramente, a experimentação, quando no caso do primeiro ou primeiros contatos com a substância. A partir dessa razão inicial, chega-se a outras motivações como o estímulo de criatividade ou facilitação de insights, relaxamento, manejo do tédio, e para aumentar o efeito de outras substâncias (Palamar, Tomas \& Kamboucos, 2015). Wright e Palfai (2012) apontam que a socialização também aparece como um dos motivos, sendo as metas de vida dos que fazem uso dessa substância no decorrer da graduação mais voltadas para projetos sociais e envolvimentos em grupos e organizações. Para os autores, os indivíduos que apresentam metas mais voltadas para carreiras acadêmicas tendem a fazer um uso menor ou nenhum uso.

Em estudo realizado por Suerken et al. (2014) é possível constatar que o meio universitário é um ambiente que facilita o acesso e a experimentação inicial de maconha e derivados, sendo que 
aproximadamente $8,5 \%$ dos indivíduos que nunca haviam usado antes do ingresso passaram a usar em seu ano de calouro, e os que já faziam uso tenderam a aumentar o consumo.

Em relação ao álcool, a prevalência de consumo entre os estudantes se mantém alta, datando desde o início dos estudos na área. Além disso, muitas vezes se dá com uso concomitante com outras substâncias tanto legais quanto ilegais, e cada vez mais tem-se notado um padrão de uso episódico pesado (10 ou mais doses) entre essa comunidade, tornando importantíssimos os estudos voltados para a topologia dessa área (Kilmer, Cronce e Larimer, 2014).

Muitos estudos buscam abarcar as razões do indivíduo para uso das substâncias (Palamar, Tomas \& Kamboucos, 2015; Kuntsche et al., 2005), mas é interessante também a observação dos motivos para a abstinência, que delimita até que ponto vai o interesse do indivíduo no uso, baseado em suas consequências. Dentre as possíveis consequências, as que se mostram mais motivadoras de comportamentos de abstinência estão a perda de controle das emoções e atitudes, danos psicológicos e fisiológicos, e expectativa de baixo prazer ou divertimento com o uso, acima de razões como possíveis prejuízos legais, custos altos ou dificuldade de acessibilidade (Lauritsen, Rosenberg \& Sprague, 2017). Dentre universitários, a literatura aponta que as consequências podem ser em geral categorizadas em fisiológica, sexual, acadêmica e social (Mallet et.al., 2015).

É possível perceber, a partir dos estudos que trabalham com levantamentos e caracterização do uso de substâncias, que se considera o uso como algo negativo que ameaça valores políticos, econômicos e sociais (Silva, Malbergier, Stempliuk \& Andrade, 2006), o que contribui para a estigmatização do mesmo. Estabelece-se uma relação clara entre o consumo de substâncias, principalmente o álcool, e a expectativa de comportamentos de risco na população universitária, além de outros fatores negativos, como queda do rendimento acadêmico, danos a patrimônio, prejuízo no desenvolvimento de habilidades cognitivo-comportamentais, dentre outros (Peuker, Fogaça \& Bizarro, 2006).

Dentro dessa dinâmica, os estudos que procuram examinar a questão do maior uso de substâncias nessa etapa da vida, tendem a ser voltados para medidas que procurem a diminuição do consumo (Lange et al., 2002), uma vez que é considerado que essa conduta aumenta a ocorrência de comportamentos de risco dos indivíduos, como dirigir embriagado ou sob efeito de outras substâncias, envolvimento em brigas com amigos e/ou polícia, e relações sexuais sem proteção (Pillon, O’Brien \& Chavez, 2005).

\subsection{Redução de Danos}

O uso de substâncias se faz presente ao longo do desenvolvimento histórico humano, sendo, portanto, um fato constante com o qual a sociedade sempre teve que lidar (Dalla Déa et al., 2004). A partir disso, com o desenvolvimento de técnicas e tecnologias mais apuradas, as substâncias 
tornaram-se acessíveis e ganharam um valor mais social, ao contrário do significado religioso ritualístico mais comum na antiguidade, levando a um consumo mais amplo. Com isso, inicia-se uma abordagem voltada para a segurança e Justiça com relação ao uso, abordagem essa com um caráter inicial majoritariamente repressivo e proibicionista (Machado \& Boarini, 2013). Essa abordagem ainda perdura nos dias de hoje, sendo caracterizada como baseada num modelo moral e criminal, promovendo muitas vezes o encarceramento do usuário e considerando o mesmo como portador de uma doença biológica, deixando assim em segundo plano a prevenção de uso e a compreensão do contexto em que se dá (Alves, 2009).

Desenvolve-se então políticas públicas pautadas na repressão, levando a uma intolerância ampla ao uso de drogas. Cobra-se do usuário que busca ajuda para lidar melhor com seu uso, uma postura de abstinência, não tendo espaço no sistema de saúde para aquele que não consegue ou não deseja cessar seu uso, formando-se ainda um estigma sobre o mesmo. Apesar dessa rigidez exacerbada e busca por uma sociedade "livre de drogas", as políticas pautadas nesses princípios não tem sua eficácia comprovada, uma vez que o consumo em escala mundial continua a aumentar, gerando mais usuários excluídos dos programas de saúde por não se enquadrarem nos requisitos (Machado \& Boarini, 2013).

Paralelamente às medidas proibicionistas que foram mais adotadas nas formas de políticas públicas de saúde, existiam também, mas com menos espaço e, portanto, menor contato com a população, as estratégias de redução de danos. Essas estratégias começaram a ganhar mais força e atenção após movimentos sociais de usuários da década de 80 , que buscavam uma melhor condição de saúde pública para os usuários. Somado a esses movimentos, havia a impossibilidade de se ignorar o crescente número de casos de AIDS ao redor do mundo, tornando-se um problema de saúde mundial (Alves, 2009).

A redução de danos teve sua origem oficialmente em 1926, com os Relatórios Rolleston que recomendavam que médicos prescrevessem ópio aos dependentes com o intuito de que os mesmos pudessem ter uma vida mais estável e adaptada socialmente. Apesar de antiga, essas estratégias não tem uma conceituação clara e convergente na literatura, sendo melhores definidas pelos seus objetivos e práticas, que buscam minimizar riscos e prejuízos psicossociais, biológicos e econômicos provindos ou secundários ao uso de substâncias, sem necessariamente buscar a abstinência das mesmas. As ações nesse âmbito são guiadas pelos princípios do pragmatismo, da diversidade e da tolerância. Deve ser pragmática no sentido de manter seus objetivos claros, voltados para a diminuição dos males e riscos provindos do uso de substâncias, se colocando como uma estratégia de saúde pública que busca preservar a vida dos usuários que não conseguem ou não desejam parar seu consumo, protegendo-os tanto de possíveis infecções e doenças, como de situações de risco que possam surgir a partir de estados alterados de consciência. Busca basear-se 
na diversidade uma vez que o consumo de substâncias apresenta essa característica, devendo então haver a mesma diversidade de estratégias, para que possa ser contemplado o maior número de usuários possíveis em sua abrangência de uso. Dessa forma, podem ser citadas como estratégias de redução de danos a troca de seringas usadas por novas, limpeza de seringas, terapias de substituição de substâncias por outras menos danosas (substituição do crack pela maconha), sempre ter um membro do grupo que não beba para poder dirigir ("motorista da rodada"), usar meios de transporte confiáveis que não necessitem da pessoa alterada dirigir (táxi, Uber), buscar condições seguras e limpas de uso, dentre outras. A redução de danos é guiada também pela tolerância no sentido do respeito ao usuário no seu direito pela sua droga de consumo, direito esse muitas vezes embasado na legislação, que por questões de preconceito e foco exacerbado na repressão a qualquer consumo muitas vezes é ignorado ou até punido indevidamente (Andrade, 2004).

O Reino Unido é um dos primeiros países a adotar uma política mais pautada na Redução de Danos, baseada nos Relatórios Rolleston. Mais recentemente, adota-se também uma estratégia integrada para abordar o uso, buscando estratégias de atuação em campo, aproximando os cuidados de saúde do usuário sem muitas exigências feitas a ele, o que vem resultando em uma maior atenção desse usuário às suas necessidades, uma vez que dessa forma os programas se tornam mais convidativos do que impositivos. Facilita-se também o acesso dessas pessoas para as próprias estratégias de redução de danos, como a troca de seringas e as terapias de substituição de substância. Aliado a isso, mantém-se uma fisscalização sobre o tráfico, principalmente sobre substâncias mais nocivas e danosas à saúde como a heroína e a cocaína (Fonseca \& Bastos, 2005).

No Brasil, a Redução de Danos começou a ser utilizada também motivada por um crescimento dos casos de AIDS decorrente do uso indevido de substâncias injetáveis. Teve seu início no município de Santos, em 1989, para em 2003, deixar de fazer parte somente de programas de DST/AIDS para se tornar uma referência no cuidado daqueles que fazem uso de álcool e outras substâncias (Passos \& Souza, 2011). Ainda assim, apesar de mostrar bons resultados em diversas iniciativas e programas, as portarias do Ministério da Saúde que normatizam as políticas relativas aos cuidados com álcool e outras drogas ainda não estruturam as políticas de Redução de Danos, se restringindo somente a publicações descritivas e propositivas, não dando atenção ao monitoramento e avaliação de programas que já vem mostrado resultados (Inglez-Dias, Ribeiro, Bastos e Page, 2014).

O Ministério de Saúde brasileiro busca uma abordagem mais pedagógica e acolhedora da questão do uso de substâncias, desenvolvendo campanhas de cunho educativo voltadas para o uso e suas implicações. Busca-se, implementar medidas que procurem informar, e a partir disso reduzir a demanda de uso e os danos acarretados pelo consumo (Fonseca \& Bastos, 2005). Deve-se ressaltar que essas medidas se dão majoritariamente no aspecto teórico, uma vez que na prática o que é visto 
ainda é uma postura de repressão e preconceito por parte das autoridades e população em geral (Machado \& Boarini, 2013).

\subsection{A Redução de Danos no contexto universitário}

Como descrito anteriormente, o consumo de substâncias no cenário universitário tem alta frequência, aumentando as chances de consequências negativas causada por esse uso. Entretanto, a literatura aponta que o universitário também desenvolve modos de ação para evitar essas consequências adversas (Frank, Thake \& Davis, 2012).

Esses modos de ação são descritos na literatura como "protective behaviors strategies" (PBS), que seria estratégias que o indivíduo pratica como intuito de reduzir os possíveis danos causados pelo consumo (Martens et.al., 2005). Assemelham-se, em conceito e atitudes, com o que apresentamos aqui como estratégias de redução de danos. Essas estratégias moderam a forma de fazer o uso de uma substância, de forma a diminuir as possíveis consequências negativas decorrentes desse consumo. Essa relação se mostra positiva no sentido de que quanto maios a presença e engajamento em PBS, menores e menos frequentes vem sido os danos dentre a população universitária, considerada uma população de alto risco de consequências negativas pelo seu padrão de uso (Martens et. al., 2011; Pearson, D’Lima e Kelley, 2013). Essa relação se mostra de outra forma: a menor frequência em que estudantes que fazem um uso pesado utilizam as estratégias de redução de danos, acumulando assim um número maior de consequências negativas do consumo, tanto em quantidade como em intensidade (Walters et.al., 2007).

Ao realizar o levantamento bibliográfico para formulação desse estudo, pode-se notar que, geralmente, quando se estudam os PBS usa-se algum instrumento que determina quais seriam as atitudes voltadas para esse cuidado, não havendo estudos que examinem quais atitudes os estudantes consideram como protetivas. Nesse sentido este trabalho se insere nessa lacuna, uma vez que será focado justamente nas percepções e vivências dos universitários.

As estratégias de redução de danos também podem aparecer como forma de moderar dificuldades em regulação do consumo. A relação de causalidade entre dificuldades de regulação e consequências negativas decorrentes do consumo aparece muito mais acentuada dentre aqueles que tem dificuldades em regular-se e não fazem uso dessas estratégias. Esse fato demonstra que essas estratégias podem ser de grande importância para os indivíduos que não conseguem ou não querem regular seu consumo, como uma alternativa para a diminuição de possíveis danos (D’Lima, Pearson e Kelley, 2012).

No caso do álcool, especificamente, outro fator que mostra relação com a frequência dos danos decorrentes do consumo são as expectativas de consumo, preditoras da forma de consumo a ser realizada, uma vez que são as expectativas de resultado daquele consumo pontual, podendo ser 
positivas ou negativas. Tanto no caso de expectativas de consumo positivas quanto negativas, as PBS aparecem como um fator importante na diminuição das consequências negativas do consumo, e, em alguns casos, também na diminuição do consumo em si (Grazioli et. al., 2014).

Dentro dos grupos de universitários também é possível notar toda uma série de comportamentos voltados para a proteção ao se dar o consumo de bebidas alcoólicas focados na imposição de limite, modo de beber e redução de danos, como definido no trabalho realizado por Frank, Thake \& Davis (2012). Os autores puderam observar que no caso da imposição de limites, há atitudes como não beber mais que um determinado número de copos, sair da festa ou bar em uma hora pré-determinada, beber água enquanto consome álcool, dentre outros; relacionado ao modo de beber, os estudantes buscam não misturar bebidas diferentes, evitar "drinking games", evitar tomar "shots", beber devagar, e não tentar acompanhar ou beber mais que os outros; já em relação ao que eles colocam como redução de danos estão os comportamentos de se manter perto de seus amigos, selecionar um "motorista da rodada" (designated driver), e saber onde estão seus copos a todo momento.

Os universitários apresentam comumente uma forma de uso de álcool conhecida como "binge drinking", em que ocorre um consumo pesado e pontual, que está mais associado a resultados negativos, como hospitalizações decorrentes do consumo excessivo e acidentes automobilísticos (Hallgren, Leifman \& Andréasson, 2012). Estudo realizado com jovens estudantes suecos concluiu que o uso de álcool mais frequente, entretanto em menor quantidade, ao invés de episódios pontuais de consumo pesado, resulta em uma diminuição dos prejuízos reportados pelos usuários (Thor, Raninen \& Landberg, 2017). Esse tipo de uso frequente e em menor quantidade pode ser considerado estratégia para diminuição de possíveis danos, uma vez que mesmo não ocorrendo a abstinência, há uma queda considerável dos episódios em que ocorre um prejuízo do indivíduo envolvido ou de terceiros.

Outro modo de formulação de estratégias voltadas para a redução de consequências negativas causadas pelo consumo de álcool se dá por meio de intervenções educativas, como manuais educativos sobre os efeitos da substância no corpo do indivíduo. Universidades americanas já desenvolveram projetos de intervenção, como o “High-Risk Drinkers project”, uma iniciativa da University of Washighton na década de noventa que buscou observar a eficiência de intervenções com universitários que faziam uso pesado de álcool. A partir desse projeto foram elaborados os programas Alcohol Skills Training Program (Fromme, Marlatt, Baer e Kivlahan, 1994) e o Brief Alcohol Screening and intervention for College Students (BASICS) (Dimeff, Baer, Kivlahan e Marlatt, 1999). Ambos os programas são alinhados com ideias de redução de danos, sendo o primeiro em pequenos grupos de alunos com apresentações sobre os efeitos, possíveis danos e treino de habilidades, enquanto o segundo se baseava numa entrevista individual protocolada que é 
adaptada para as peculiaridades do aluno buscando informar-lhe e fazer-lhe refletir sobre seu consumo, mostrando resultados positivos em suas aplicações (Marlatt e Witkiewitz, 2002).

Os resultados dessa forma de abordagem podem ser vistos no trabalho realizado por Silva e Tucci (2015), em que os estudantes que mostraram um uso de maior risco e intensidade participaram de uma intervenção pautada em informações educativas e apresentação de alternativas ao padrão de consumo atual, levando a um consumo mais consciente e menos danoso.

Mallet et.al. (2015) ao realizar uma revisão sistemática da literatura relativa às intervenções voltadas para diminuição do consumo e consequências negativas em relação ao álcool, aponta que dentre as formas de intervenção aplicadas em contextos de universidade, as abordagens informativas tendem a ser menos efetivas do que intervenções que são voltadas para atitudes e estratégias. Dentre as mais efetivas se encontra a entrevista motivacional, que procura trabalhar com quatro processos fundamentais desenvolvidas através do engajamento, foco, evocação e planejamento, buscando dar autonomia ao indivíduo quanto a estabelecer sua meta de consumo (podendo ser a abstinência ou não), e ajuda-lo de forma empática e não impositiva a atingir essa meta (Figlie e Guimarães, 2014).

Todas essas formas de se proteger das consequências negativas potenciais do uso se mostram úteis entre estudantes universitários, principalmente em situações em que estes são mais vulneráveis, como entre aqueles que apresentam sintomatologia ansiosa e depressiva ou algum outro transtorno psiquiátrico, e também em situações onde há desigualdade de gênero (Kenney \& LaBrie, 2013).

Alguns estudos abordam a questão relativa ao gênero. Estudo realizado descreveu estratégias adotadas por mulheres estudantes universitárias que já tiveram algum histórico com assédio sexual para se protegerem de possíveis assédios quando estão fazendo uso de álcool. Dentre estas, destacam-se planejar com antecedência o modo como se deslocarão na ida e na volta ao local onde irão consumir bebidas alcoólicas, andarem sempre acompanhadas de amigos confiáveis e estarem atentas aos seus copos, de modo a não terem suas bebidas adulteradas (Gilmore, et al., 2015).

As estratégias usadas por mulheres podem ser separadas em relacionadas a um uso controlado (não beber mais que um determinado número de doses e beber devagar) e formas de reduzir os danos em relação a encontros sexuais inesperados (andar sempre acompanhada, estar com pessoas de confiança). Essas estratégias resultam em um menor índice de situações de assédio ou agressão sexual (ex. homem usar de força para fazer sexo, interpretar erroneamente o interesse da mulher em fazer sexo), mas não se mostram tão eficazes em relação a episódios de comportamento sexual de risco (ex. não usar proteção, fazer sexo com alguém que normalmente não faria) conforme afirmam Moorer et al., (2013). O mesmo estudo também indica que as 
mulheres universitárias tendem a utilizar muito mais as estratégias de redução de danos do que as voltadas para controle de consumo, ressaltando a importância dessas estratégias na moderação da relação de uso.

As estratégias de redução de danos entre estudantes também colaboram para a diminuição do estigma voltado para o uso e o usuário de drogas. Estudo realizado por Barbour, McQuade \& Brown (2017) analisou situações em que estudantes ajudavam em instituições de troca e organização de agulhas, identificando que isto proporcionava um aprendizado da realidade do uso de substâncias e também de possíveis estratégias a serem usadas por eles mesmos caso consumissem alguma substância. Os autores concluem que ao se inserir e melhor compreender o meio e as estratégias, há uma diminuição do estigma em relação à busca de ajuda e pode-se aprimorar as próprias estratégias, uma vez que o uso acontece. Nesse sentido, aproxima-se o meio universitário da política de redução de danos, colaborando para que a mesma permeie o uso em si.

Atualmente, estudantes cuja formação esta relacionada ao tratamento ou à interação com indivíduos que fazem uso de substâncias tem uma maior aceitação de metas relacionadas a nãoabstinência, dependendo do grau de severidade do uso e da substância em questão, sendo possível notar que há uma maior tolerância e uma diminuição do preconceito em relação ao uso, apesar de não se aplicar a todas as substâncias. Isso é o que aponta o trabalho de Davis \& Lauristen (2016), em que 37\% dos estudantes consideravam aceitável uma meta final de não-abstinência para pacientes com transtornos de uso relacionado à cannabis, e 31\% quando relacionado ao álcool, enquanto para outras drogas ilícitas (hard drugs) apenas 19\% considerava o trabalho não focado na abstinência. Mesmo tendo um índice menor de aceitabilidade, estes dados mostram uma maior tolerância em relação às chamadas "hard drugs", quando comparado com estudos realizados anteriormente. $\mathrm{O}$ estudo considera que esse desenvolvimento e redução do preconceito quanto ao uso são resultados de um maior contato durante a formação com os conceitos da Redução de Danos, que permitem a esses estudantes entenderem que o consumo (que inclusive eles mesmos fazem) não precisa necessariamente ser alvo de repressões e preconceitos, desde que possa ser manejado de forma a não diminuir a qualidade de vida e saúde do indivíduo.

\section{CONCLUSÕES}

Pudemos perceber ao longo da exposição do que foi trabalhado nesse estudo que os universitários apresentam um padrão de uso de risco para múltiplas substâncias, sendo um recorte da sociedade caracterizado por esse consumo (SENAD, 2010), e, portanto, mais aberto as possíveis consequências negativas desse uso (Martens et. al., 2011; Pearson, D’Lima e Kelley, 2013). Dentro desse consumo foi possível uma perspectiva das percepções dos próprios estudantes acerca de suas vulnerabilidades, sendo esses dados de suma importância para a compreensão deste contexto de uso como um todo. 
Pudemos explicitar melhor os detalhes da entrada do estudante no ambiente universitário e os impactos que isso apresenta em sua vida tanto no tocante ao seu uso de substâncias. É notável como o ambiente universitário aparece como um facilitador de acesso e também motivador de consumo (Suerken et al., 2014), sendo essas características percebidas e descritas pelos participantes ao longo das entrevistas. São descritas, com concordância com a literatura (Laranjo \& Soares, 2006; Fachini \& Furtado, 2013; Garrido, 2015) as razões para essa vulnerabilidade, relacionadas a uma maior liberdade do indivíduo no ambiente universitário, em comparação à casa dos pais.

A maior liberdade descrita pelos participantes acontece concomitante com percepções acerca da cultura de consumo universitária, que é descrita como um fator que incentiva o consumo. Esse incentivo se dá pelos estudantes terem expectativas positivas sobre o consumo (Kunstche et al., 2005) incentivadas pelo seu contexto, onde as festas e valorização da vida social colaboram para uma estímulo ao uso de substâncias (Mallett et al., 2015). Aliado a isso está um fator que os estudantes colocam que não se encontra tão consolidado na literatura, relativo a uma dificuldade em conversar abertamente sobre o próprio consumo, fator esse que dificulta o processo do indivíduo em se adaptar ao novo meio. Chama a atenção a questão da grande maioria dos estudantes afirmar nunca ter tido aquele tipo de conversa antes, referindo-se às entrevistas, demonstrando o quanto os diálogos no campo do uso de substâncias são cercados por uma aura de preconceito e imposição, muito incentivada pelas posturas governamentais e sociais de "guerra às drogas" (Machado e Boarini, 2013).

O contexto universitário mais influenciado pela cultura de uso descrito pelos participantes é o contexto de república universitária. Devido ao método de seleção de participantes, foi possível ao presente estudo o contato com muitos jovens desse meio, cujos dados carecem na literatura. Este fato por si só já demonstra a complexidade do meio universitário, constituído por grupos heterogêneos de estudantes, que fazem parte de diversos contextos dentro desse meio (Kilmer, Cronce e Larimer, 2014). Dentro das vivências em república, destaca-se que há um uso corriqueiro no dia-a-dia, o que incentiva ainda mais o uso através da busca pela inserção social naquele meio, levando muitas vezes o estudante a se submeter a esse padrão de uso (Matos e Projeto Aventura Social, 2010). Porém, se isso é visto pelo estudante como um fator que tende a aumentar sua vulnerabilidade, a república também traz um aspecto de rede de proteção, uma vez que os integrantes apresentam preocupações em se cuidar e proteger entre si. Essa rede de proteção é comum a todas as repúblicas, mas mais intensa dentre as repúblicas femininas, vide a maior percepção de vulnerabilidade das mulheres dentro dos contextos de uso (Fachini \& Furtado, 2013; Gilmore et al., 2015). 
Descrevemos também as consequências negativas relatadas pelos estudantes decorrentes de seu consumo, às quais puderam ser separadas em quatro tipos: financeiras, acadêmicas, físicas e psicossociais. Essa classificação se diferencia da literatura, que aponta para consequências sexuais, e não coloca consequências financeiras (Mallett et al, 2015). As consequências sexuais descritas foram inseridas em consequências psicossociais, pois os relatos descreveram-nas como muito mais impactantes nesse aspecto da vida dos estudantes. Essa consequências foram abordadas considerando muito mais os impactos que poderiam ter em como o jovem se sente e se coloca após algum ocorrido, do que em possíveis prejuízos de se contrair doenças ou gravidez inesperada, aspectos mais contemplados na bibliografia da área (Gilmore et al, 2015). Dentre todos os tipos de consequências, os que tiveram maior destaque no sentido de mais impactar a vida do estudante foram os danos psicossociais, demonstrando mais uma vez a importância de como o jovem se relaciona com seus pares e lida com o que sente nessa etapa da vida (Laranjo \& Soares, 2006).

Foi percebido que a maioria dos estudantes têm uma noção da necessidade das medidas protetivas relativas ao uso que fazem, seja esse no contexto de festas "open bar", bares pagos, ou dentro do convívio em república ou com os amigos. Nesse aspecto, a diferença notável em comparação com a literatura (Gilmore et al., 2015; Frank, Thake \& Davis 2012) é a dificuldade apresentada e relatada por nossos participantes de estabelecer limites para seu consumo, sendo as estratégias relativas a esse comportamento mais prejudicadas e menos elaboradas. Quando em uma situação que o consumo deve ser controlado, eles demonstraram preferir não consumir, ou melhor, não ir para ambientes onde se dá o consumo, uma vez que a pressão social e o desejo de consumir dificultam o manejo.

É possível compreender que o primeiro fator para o estudante perceber essa necessidade de cuidado dentro de seu uso, e começar a buscar forma de desenvolver medidas protetivas, se dá através da percepção de suas vulnerabilidades e principalmente da cultura de uso universitária. É nesse ponto em que os dois manuscritos desse estudo convergem, pois ao final do processo de percepção da vulnerabilidade e das consequências às quais está vulnerável que se inicia o processo de desenvolvimento das estratégias de redução de danos.

Essa percepção acarreta um estímulo ao aprendizado, e esse aprendizado não se distancia do que coloca Bandura (1971) acerca de sua teoria de aprendizagem social, em que o indivíduo é capaz de modificar seu comportamento através da observação de seus pares, e das experiências individuais. No caso dos estudantes, isso se traduz em sentir alguns dos danos decorrentes do consumo, e a partir daí valorizar formas evita-los, ou observar seus pares vivendo ou lidando com esses danos. 
Deve-se ressaltar que os jovens em alguns momentos tentam desvalorizar os danos sofridos no sentido de fazer piadas com eles, principalmente perante seus pares. Porém há uma contradição nesse ponto, uma vez que ainda consideram as consequências que viveram como negativas. Sendo assim, valorizam as formas de aprendizado através do diálogo e vivência com os pares, ressaltando seus veteranos como importantes colaboradores desse processo. Há uma demanda dos estudantes por diálogos abertos sobre uso de substâncias, uma vez que têm a percepção da uiversidade não estimular esse diálogo (Amaral, Lourenço e Ronzani, 2006). Esse pode ser um indicativo de uma possível brecha para intervenção a ser examinada por futuros estudos nessa área, vide que a própria entrevista em grupo focal já foi suficiente para gerar reflexão nos participantes. Nesse sentido, o discurso dos participantes valoriza diálogos e posturas não impositivas e compreensivas em relação ao uso, sendo esse o tratamento que percebem em seus pares (Figlie e Guimarães, 2014).

O principal foco das estratégias de redução de danos construídas e mantidas pelos estudantes são os eventos festivos, um grande cerne da cultura universitária como um todo (Marzell et al., 2015). As festas são categorizadas em festas modelo open bar, onde as bebidas são servidas sem custos no decorrer da festa inteira, e as festas modelo bar vendido, onde as bebidas são vendidas ao longo do evento, que geralmente tem duração menor. A partir do evento, os estudantes buscam formas de se preparar buscando evitar prejuízos, modos de ir e voltar sem correr riscos, maneiras de agir no decorrer do evento que evitem danos, e atitudes a se tomar nos dia seguinte ao evento para se recuperar e lidar com qualquer dano sofrido.

Suas estratégias de redução de danos não fogem das descritas na literatura (Davis \& Lauristen, 2016; D’Lima, Pearson \& Kelley, 2012; Kenney \& LaBrie, 2013), valorizando a alimentação antes do evento, evitar dirigir enquanto embriagado, buscar tomar água no decorrer do evento, e procurar o apoio de amigos em casos de dificuldades em qualquer momento. Esta última estratégia é a que mais valorizam, reforçando o valor que as redes de proteção que os jovens formam entre si podem ter.

O presente estudo teve como objetivo compreender as percepções que estudantes universitários que fazem uso de drogas têm sobre seu consumo, e como se constroem suas estratégias de redução de danos para lidar com o mesmo. Um dos aspectos de maior valor a ser ressaltado é o da ênfase nas experiências e vivências dos estudantes, buscando compreender sob sua ótica o contexto universitário, valorizando o ponto de vista do usuário.

Os resultados demonstraram que os próprios estudantes entendem que dentro de seu padrão de consumo se colocam em muitas situações de vulnerabilidade, demonstrando uma percepção de sua própria cultura como um fator de incentivo e que muitas vezes dificulta o indivíduo ter o 
autocuidado apropriado. Pode-se perceber o quanto o uso dentro do contexto universitário é esperado e incentivado, mas de certa forma ainda se coloca como um tabu, uma vez que não é discutido nem existe na universidade uma abertura para que os estudantes possam se informar sem ser condenados ou julgados. Nesse sentido podemos ver um papel ambivalente da república universitária na forma como ela se relaciona com as vulnerabilidades e com a proteção, baseado em seu padrão de uso mais intenso e frequente, ao mesmo tempo em que se mostra uma entidade que forma uma rede de proteção para o indivíduo, sendo muitas vezes o local ondo ocorrem os diálogos sobre o uso e o aprendizado com os pares. Descreveram-se os principais danos e seus tipos a partir da ótica do estudante, em contraponto a maioria dos estudos da área, permitindo uma reflexão acerca das medidas protetivas pertinentes a esse meio a partir da perspectiva de quem vive nele. Buscou-se essa perspectiva também ao se procurar entender como as estratégias de redução de danos se formavam, e quais as principais orientações das mesmas. Com base nisso, apesar de não haver nenhuma estratégia que fuja muito do que já é conhecido, foi possível organizá-las de acordo com o que os estudantes valorizaram, permitindo a adaptação das mesmas aos diferentes momentos dos eventos festivos onde o consumo é esperado e maior.

O estudo em questão foi capaz de examinar somente uma população universitária, no sentido da maioria dos estudantes pertencerem quase todos ao mesmo campus, apesar do convite de participação ter sido direcionado à diferentes universidades da cidade. Uma das limitações do estudo se dá por conta da metodologia de seleção de participantes, que acarretou em um foco em grupos de estudantes que podem não corresponder integralmente à população como um todo. Apesar disso, esse fato também apresentou um ganho por permitir acesso à um grupo pouco explorado pela literatura, os moradores de república universitária brasileira, contribuindo com dados pertinente a esse grupo específico. O outro limite a ser considerado é o fato de que as estratégias apresentadas, por mais que também encontradas na literatura, talvez não sejam capazes de abarcar e fazer sentido a todos os estudantes, até pela própria natureza pessoal do desenvolvimento de estratégias de redução de danos. Ainda assim, é de suma importância documentar as estratégias mais gerais uma vez que foi percebido um déficit no conhecimento dos estudantes acerca de medidas protetivas em relação ao consumo.

Trazemos contribuições à área principalmente no tocante a compreensão dos contextos de uso universitário a partir do próprio estudante. Essa compreensão é de vital importância para qualquer universidade que busque um diálogo de maior qualidade acerca do assunto com seus alunos. Não se pode, ao se tratar do assunto, buscar impor formas de cuidado que não façam sentido à população a qual essas formas são direcionadas. Ainda foi possível um indicativo de uma iniciativa entre os estudantes de formação de uma rede de proteção, onde há uma preocupação dos 
jovens em cuidar de seus pares, e onde o diálogo parece se dar de forma mais acessível e que faça mais sentido para eles. Dentro dessa rede, é possível perceber que um dos principais fatores que a torna valorizada perante o jovem, é a característica dos indivíduos nela manterem posturas empáticas e sem julgamentos, havendo um cuidado para momentos de embriaguez, e uma disponibilidade para conversas caso ocorra algum dano ou prejuízo. Nesse aspecto, o trabalho pode demonstrar o papel que a empatia e honestidade podem ocupar ao se tratar de assuntos relativos ao uso de substâncias, podendo esse tipo de abordagem ser uma arma valiosa para tornar o indivíduo apto e capaz de definir para si seus padrões de uso, baseado em suas características, reflexões, desejos e peculiaridades.

Visando maiores ganhos para a área de conhecimento a qual dedicamos o presente estudo, sugerem-se maiores estudos com um foco na percepção dos estudantes, sem procurar focar em estratégias ou conhecimentos pré-definidos. Para tais trabalhos, seria interessante a postura empática e não impositiva que foi valorizada pelo grupo estudado, para uma maior compreensão de como as universidades podem começar a estabelecer diálogos com seus estudantes de forma a estabelecer uma relação de parceria. Seria também um ganho nesse sentido estudos buscando compreender como se desenvolvem e funcionam as redes de proteção criadas entre os estudantes, de forma a incentivar a criação de redes saudáveis onde as informações compartilhadas são de confiança e tem validade na prática. Para tais estudos, é interessante examinar a população de moradores de república universitária, permitindo um conhecimento acerca das diferentes redes de proteção que podem ser formadas em diferentes contextos universitários. 



\section{- REFERÊNCIAS}

Acharya, L., Jin, L., \& Collins, W. (2018). College life is stressful today - Emerging stressors and depressive symptoms in college students. Journal of American College Health, 1-10.

Alves, V. S. (2009). Modelos de atenção à saúde de usuários de álcool e outras drogas: discursos políticos, saberes e práticas. Cadernos de Saúde Pública, 25(11), 2309-2319

Amaral, M. B., Lourenço, L. M., \& Ronzani, T. M. (2006). Use among University Students. Journal of Substance Abuse Treatment, 31, 181-185

American Psychiatric Association (2000). Diagnostic and statistical manual of mental disorders. 4th ed. revised. Washington, DC: American Psychiatric Association.

Andrade, T. M. (2004). Redução de danos: um novo paradigma?. Tempos, lugares $e$ olhares sobre seu consumo, 87.

Antoniassi, G.J., Meneses-Gaya C. (2015). Implicações do uso de álcool, tabaco e outras drogas na vida do universitário. Rev Bras Promoção Saúde. 28(1): 67-74.

Bandura, A. (1971). Social Learning Theory. General Learning Corporation. Stanford University, New York.

Baskhar, R. (2014). The Order of Natural Necessity: A Kind of Introduction to Critical Realism. University College London: Institute of Education.

Brasil. Presidência da República. Conselho Nacional de Saúde. Resolução Número 466. Brasília: Dezembro de 2012. 
Brasil. Presidência da República. Secretaria Nacional de Políticas sobre Drogas. I Levantamento Nacional sobre o Uso de Álcool e Outras Drogas entre Universitários das 27 Capitais Brasileiras. Brasília: SENAD; 2010.

Braun, V. \& Clarke, V. (2006). Using Thematic Analysis in Psychology. Qualitative Research in Psychology. 3, 77-101.

Braun, V. \& Clarke, V. (2013). Successful Qualitative Research: a practical guide for begginers. Sage: London.

Braun V. \& Clarke V. (2019). Reflecting on reflexive thematic analysis. Qualitative Research in Sport, Exercise and Health, 11:4, 589-597.

Carlini, E.A.; Galduróz, J.C.F.; Noto, A.R.; Fonseca, A.M.; Carlini, C.M.; Oliveira,L.G.; Nappo S.A.; Moura, Y.G.; Sanchez, Z.V.M. (2005). II Levantamento Domiciliar sobre o uso de Drogas Psicotrópicas no Brasil: estudo envolvendo as 108 maiores cidades do país. - 2005. São Paulo: CEBRID - Centro Brasileiro de Informações sobre Drogas Psicotrópicas, Departamento de Psicobiologia, UNIFESP - Universidade Federal de São Paulo.

Carlini, E. L. A., Noto, A. R., Sanchez, Z. V. D. M., Carlini, C. M. A., Locatelli, D. P., Abeid, L. R., et al.(2010). VI Levantamento Nacional sobre o Consumo de Drogas Psicotrópicas entre Estudantes do Ensino Fundamental e Médio das Redes Pública e Privada de Ensino nas 27 Capitais Brasileiras -2010. São Paulo: CEBRID - Centro Brasileiro de Informações sobre Drogas Psicotrópicas: UNIFESP - Universidade Federal de São Paulo. Brasília: SENAD - Secretaria Nacional de Políticas sobre Drogas.

Conselho Federal de Psicologia (2005). Código de Ética Profissional do Psicólogo.

Cotrin, B.C. (1996). Potencialidades da técnica qualitativa grupo focal em investigações sobre abuso de substâncias. Rev. Saúde Pública. 30 (3): 285-93

Dalla Déa, H.R.F., Santos, E.N., Itakura, E. \& Olic, T.B. (2004). A Inserção do Psicólogo no Trabalho de Prevenção ao Abuso de Álcool e Outras Drogas. Psicologia: Ciência e Profissão. 24 (1), 108-115

Davis, A.K. \& Lauritsen, K.J. (2016). Acceptability of nonabstinence goals among students enrolled in addiction studies programs across the United States. Substance Abuse. 37:1, 204-208

Dennhardt, A.A. \& Murphy, J.G. (2013). Prevention and treatment of college student drug use: A review of the literature. Addictive Behaviors. 38, 2607-2618. 
Derefinko, K.J., Charnigo, R.J., Peters, J.R., Adams, Z.W., Milich, R., \& Linam, D.R. (2016). Substance Use Trajectories From Early Adolescence Through the Transition to College. $\underline{J}$ Stud Alcohol HYPERLINK "https://www.ncbi.nlm.nih.gov/pubmed/27797694"_HYPERLINK HYPERLINK

"https://www.ncbi.nlm.nih.gov/pubmed/27797694"'"https://www.ncbi.nlm.nih.gov/pubmed/2779769 4 HYPERLINK "https://www.ncbi.nlm.nih.gov/pubmed/27797694"" _ HYPERLINK "https://www.ncbi.nlm.nih.gov/pubmed/27797694" HYPERLINK "https://www.ncbi.nlm.nih.gov/pubmed/27797694" HYPERLINK HYPERLINK "https://www.ncbi.nlm.nih.gov/pubmed/27797694"'"https://www.ncbi.nlm.nih.gov/pubmed/2779769 4 HYPERLINK "https://www.ncbi.nlm.nih.gov/pubmed/27797694"" _ HYPERLINK "https://www.ncbi.nlm.nih.gov/pubmed/27797694"Drugs HYPERLINK_HYPERLINK "https://www.ncbi.nlm.nih.gov/pubmed/27797694"'"https://www.ncbi.nlm.nih.gov/pubmed/2779769 4_HYPERLINK_ "https://www.ncbi.nlm.nih.gov/pubmed/27797694"" I HYPRLINK "https://www.ncbi.nlm.nih.gov/pubmed/27797694". 77(6), 924-935.

Dimeff, L. A., Baer, J. S., Kivlahan, D. R., \& Marlatt, G. A. (1999). Brief Alcohol Screening and Intervention for College Students (BASICS): a harm reduction approach. New York, NY, USA: Guilford Press

D'Lima, G. M., Pearson, M. R., \& Kelley, M. L. (2012). Protective behavioral strategies as a mediator and moderator of the relationship between self-regulation and alcohol-related consequences in first-year college students. Psychology of Addictive Behaviors, 26(2), 330-337.

Edland-Gryt, M. Sandberg, S. e Pedersen, W. (2017). From ecstasy to MDMA: Recreational drug use, symbolic boundaries, and drug trends. Int J Drug Policy, 50, pp. 1-8.

Fachini A. \& Furtado E.F. (2013) Uso de álcool e expectativas do beber entre universitários: uma análise das diferenças entre os sexos. Psicol Teor Pesq. 29(4), 421-428.

Fernandes, L. \& Carvalho, M.C. (2000). Por onde anda o que se oculta: o acesso a mundos sociais de consumidores problemáticos de drogas através do método do snowball. Rev. Toxicodependências. 6(3), 17-28.

Figlie, N B.;Guimarães, L P. A entrevista motivacional: conversas sobre mudança (2014). Bol. Acad. Paulista de Psicologia, São Paulo. v. 34, nº87, p. 472-489

Frank, C., Thake, J., Davis, C.G. (2012). Assessing the Protective Value of Protective Behavioral Strategies. J. Stud. Alcohol Drugs. 73, 839-843 
Fromme, K., Marlatt, G. A., Baer, J. S., \& Kivlahan, D. R. (1994). The Alcohol Skills Training Program: a group intervention for young adult drinkers. Journal of Substance Abuse Treatment, 11, $143-154$.

Garrido, E.N. (2015). A Experiência da Moradia Estudantil Universitária: Impactos sobre seus Moradores. Psicologia: Ciência e Profissão, 35(3), 726-739

Gilmore, A. K., Stappenbeck, C. A., Lewis, M. A., Granato, H. F., \& Kaysen, D. (2015). Sexual Assault History and Its Association With the Use of Drinking Protective Behavioral Strategies Among College Women. Journal of Studies on Alcohol and Drugs. 76(3), 459-464.

Goldsmith, A. A., Thompson, R. D., Black, J. J., Tran, G. Q., \& Smith, J. P. (2011). Drinking Refusal Self-Efficacy and Tension-Reduction Alcohol Expectancies Moderating the Relationship Between Generalized Anxiety and Drinking Behaviors in Young Adult Drinkers. Psychology of Addictive Behaviors. 59-67.

Gondin, S.M.G. (2003). Grupos focais como técnica de investigação qualitativa: desafios metodológicos. Paidéia. 12(24), 149-161

Grazioli, V. S., Lewis, M. A., Garberson, L. A., Fossos-Wong, N., Lee, C. M., \& Larimer, M. E. (2015). Alcohol Expectancies and Alcohol Outcomes: Effects of the Use of Protective Behavioral Strategies. Journal of Studies on Alcohol and Drugs, 76(3), 452-458.

Günther, H. (2006). Pesquisa Qualitativa versus Pesquisa Quantitativa: Esta É a Questão? Psic.:Teor. e Pesq. 22(2), 201-210.

Hallgren, M., Leifman, H. \& Andréasson, S. (2012). Drinking Less But Greater Harm: Could Polarized Drinking Habits Explain the Divergence Between Alcohol Consumption and Harms among Youth?. Alcohol and Alcoholism. 47(5), 581-590.

Henrique, I.F.S. et al. (2004). Validação da versão brasileira do teste de triagem do envolvimento com álcool, cigarro e outras substâncias (ASSIST). Revista da Associação Médica Brasileira. 50(2). São Paulo. 199-206.

Henry-Edwards S., Humeniuk R., Ali R., Poznyak V. and Monteiro M. (2003). The Alcohol, Smoking and Substance Involvement Screening Test (ASSIST): Guidelines for Use in Primary Care (Draft Version 1.1 for Field Testing). Geneva. World Health Organization. 
Inglez-Dias, A,; Ribeiro, J M;;Bastos, F I,; Page, K. (2014). Políticas de redução de danos no Brasil: Contribuições de um programa norte-americano. Ciência \& amp; Saúde Coletiva, v.19, nº 1, p 147-157.

Kenney, S.R. \& LaBrie, J.W. (2013). Use of Protective Behavioral Strategies and Reduced Alcohol Risk: Examining the Moderating Effects of Mental Health, Gender and Race. Psychol Addict Behav. 27(4): 997-1009.

Kilmer J.R., Cronce J.M. e Larimer M.E. (2014). College Student Drinking Research From the 1940s to the Future: Where We Have Been and Where We are Going. J. Stud. Alcohol and Drugs, Supplement 17, 26-35.

Kirstin J. L., Rosenberg, H. \& Sprague J.E. (2017). University students' attributions for abstinence from synthetic cannabinoids and synthetic cathinones, The American Journal of Drug and Alcohol Abuse. 1-9.

Kuntsche, E., Knibbe, R., Gmel, G., Engels, R. (2005). Why do young people drink? A review of drinking motives. Clinical Psychology Review. 25, 841-861.

Kuntsche, E., Knibbe, R., Gmel, G., Engels, R. (2006). Who drinks and why? A review of socio-demographic, personality, and contextual issues behind the drinking motives in young people. Addictive Behaviors. 31, 1844 - 1857.

Lange, J.E., Clapp J.D., Turrisi R., Reavy R.L., Jaccard J., Johnson M.B., et al. (2002) College Binge Drinking: What Is It? Who Does It? Alcoholism: Clin. Exp. Res. 26(5), 723-30.

Laranjo, T., \& Soares, C. (2006). University residence halls: socialization processes and drug consumption .Revista de Saúde Pública. 40(6), 1027-1034

Larkin, M \& Thompson, A 2012, Interpretative phenomenological analysis. in A Thompson \& D Harper (eds), Qualitative research methods in mental health and psychotherapy: a guide for students and practitioners. John Wiley \& Sons, Oxford, 99-116

Machado, L.V. \& Boarini, M.L. (2013). Políticas Sobre Drogas no Brasil: a Estratégia de Redução de Danos. Psicologia: ciência e profissão. 33 (3), 580-595.

Magnabosco, M.B., Formigoni, M.L.O.S., Ronzani, T.M. Avaliação dos padrões de uso de álcool em usuários de serviços de Atenção Primária à Saúde de Juiz de Fora e Rio Pomba (MG). Revista Brasileira de Epidemiologia, Juiz de Fora, p.637-647, 2007. 
Mallett, K. A., Turrisi, R., Cleveland, M. J., Scaglione, N. M., Reavy, R., Sell, N. M., \& Varvil-Weld, L. (2015). A Dual-Process Examination of Alcohol-Related Consequences Among First-Year College Students. Journal of Studies on Alcohol and Drugs, 76(6), 862-871.

Marlatt, G A,; Witkiewitz, K. (2002). Harm reduction approaches to alcohol use: Health promotion, prevention, and treatment. Addictive Behaviors, v. 27, p 867-886.

Martens, M. P., Martin, J. L., Littlefield, A. K., Murphy, J. G., \& Cimini, M. D. (2011). Changes in Protective Behavioral Strategies and Alcohol Use among College Students. Drug and Alcohol Dependence, 118(2-3), 504-507

Martens, M. P., Ferrier, A. G., Sheehy, M. J., Corbett, K., Anderson, D. A., \& Simmons, A. (2005). Development of the Protective Behavioral Strategies Survey. Journal of Studies on Alcohol, 66, 698-705.

Marzell, M., Bavarian, N., Paschall, M. J., Mair, C., \& Saltz, R. F. (2015). Party Characteristics, Drinking Settings, and College Students' Risk of Intoxication: A Multi-Campus Study. The Journal of Primary Prevention, 36(4), 247-258.

Matos, M., \& equipa do Projeto Aventura Social (2010). A Saúde dos Adolescentes Portugueses. Lisboa: Edições FMH.

Moorer, K.D., Madson, M.B., Mohn, R.S. \& Nicholson, B.C. (2013). Alcohol Consumption and Negative Sex-Related Consequences among College Women: The Moderating Role of Alcohol Protective Behavioral Strategies. J. Drug education. 43(4) 365-383.

Morgan, D. L. Focus group as qualitative research. London: Sage, 1997

Palamar, J.J., Tomas, M.G. \& Kamboukos, D. (2015). Reasons for Recent Marijuana Use in Relation to Use of Other Illicit Drugs among High School Seniors in the United States. Am J Drug Alcohol Abuse. 41(4), 323-331.

Passos, E.H. \& Souza, T.P. (2011). Redução de danos e saúde pública: construções alternativas à política global de "guerra às drogas". Psicologia \& Sociedade. 23 (1), 154-162.

Pearson, M. R., D’Lima, G. M., \& Kelley, M. L. (2013). Daily Use of Protective Behavioral Strategies and Alcohol-Related Outcomes Among College Students. Psychology of Addictive Behaviors : Journal of the Society of Psychologists in Addictive Behaviors, 27(3)

Peuker, A.C.; Fogaça, J.; Bizarro, L.(2006). Expectativas e beber problemático entre universitários. Psicologia: Teoria e Pesquisa. 22(2), 193-200. 
Pillon, S., O'Brien, B., \& Chavez, K. (2005). A relação entre o uso de drogas e comportamentos de risco entre universitários brasileiros. Revista Latino-Americana de Enfermagem. 13(spe2), 1169-1176.

Rostami M., Rezayof A., Alijanpour S., Sharifi K.A. (2017). Hippocampal nicotinic receptors have a modulatory role for ethanol and MDMA interaction in memory retrieval, Brain Research, 1669, 11-17.

Santos, M.L.R.R. (2011). Saúde mental e comportamentos de risco em estudantes universitários. Tese de Doutorado, Universidade de Aveiro (Portugal).

Sawyer A. (2001). Características chave do realismo crítico na prática: um breve resumo. Estudos de Sociologia, Rev, do Prog. de P ós-Graduação em Sociologia da UFPE. 6(2): 7-32

Shannon, R.E., Bradley, N.C., Teresa, H.M. (1999). Sources of Stress among College Students. College Student Journal, vol.30 (2).

Silva, E.C. \& Tucci, A.M. (2015). Intervenção Breve para Redução do Consumo de Álcool e suas Consequências em Estudantes Universitários Brasileiros. Psicologia: Reflexão e Crítica, 28(4), 728-736.

Silva, L., Malbergier, A., Stempliuk, V., \& Andrade, A. (2006). Factors associated with drug and alcohol use among university students . Revista de Saúde Pública, 40(2), 280-288

Silva, M.L.; Rego, F.S.; Roque, N.F.; Valenti, V.E. (2014). Use of psychoactive substances in students at a public university. ABCS Health Sci. 39(3),160-166.

Smith, J.A. \& Osborn, M. (2007). Interpretative Phenomenological Analysis. 53-80.

Soldera, M., Dalgalarrondo, P., Corrêa Filho, H., \& Silva, C. (2004). Use of psychotropics drugs among students: prevalence and associated social factors . Revista de Saúde Pública, 38(2), 277-283.

Suerken, C.K., Reboussin, B.A., Sutfin, E.L., Wagoner, K.G., Spangler, J., Wolfson, M. (2014). Prevalence of marijuana use at college entry and risk factors for initiation during freshman year. Addictive Behaviors. 39, 302-307.

Trad, L.A.B. (2009). Grupos focais: conceitos, procedimentos e reflexões baseadas em experiências com o uso da técnica em pesquisas de saúde. Physis: Revista de Saúde Coletiva. 19(3), 777-796. 
Tong A., Sainsbury P. \& Craig J. (2007) Consolidated criteria for reporting qualitative research (COREQ): a 32-item checklist for interviews and focus groups, International Journal for Quality in Health Care, V19 (6), 349-357.

Turato, E.R. (2005). Métodos qualitativos e quantitativos na área da saúde: definições, diferenças e seus objetos de pesquisa. Rev. Saúde Pública. 39(3), 507-514.

United Nations Office on Drugs and Crime - UNODC. Global illicit drug trends 2004. New York (NY): United Nations; 2004.

Walters, S. T., Roudsari, B. S., Vader, A. M., \& Harris, T. R. (2007). Correlates of Protective Behavior Utilization among Heavy-Drinking College Students. Addictive Behaviors, 32(11), 2633-2644.

Willig, C (2001). Introducing qualitative research in psychology: adventures in theory and method. New York - USA: Open University Press.

World Health Organization (1994). Lexicon of alcohol and drug terms. Geneva.

World Health Organization (2004). Neuroscience of psychoactive substance use and dependence. Geneva.

Yin, R. K. (2015). Estudo de caso: planejamento e métodos. (5 $5^{\mathrm{a}}$ ed.). Porto Alegre: Bookman. 